\title{
Features visualization of geometric objects in the $\mathrm{BN}$-calculus
}

\author{
E.V. Konopatskiy, ${ }^{1, A}$, A.A. Bezditnyi²,B, Ya.A. Kokareva3,C, V.V. Kucherenko,,D \\ A Donbas National Academy of Civil Engineering and Architecture \\ в Sevastopol branch of «Plekhanov Russian University of Economics» \\ C Don State Technical University \\ D The limited liability company «South-West Trading Company» \\ ${ }^{1}$ ORCID: 00oo-0oo3-4798-7458, e.v.konopatskiy@mail.ru \\ 2 ORCID: 0000-0003-0528-9731, pereverten_1985@mail \\ 3 ORCID: oooo-0003-4408-6777, yanka demon@rambler.ru \\ 4 ORCID: 0000-0003-2057-3060, drago magic@mail.ru
}

\begin{abstract}
The article discusses an approach to imaging of geometric objects based on point equations of their formation. Spot equations that are represented in symbolic form, are reduced to a system of parametric equations using coordinate-wise calculation. The number of equations of the system depends on the dimension of the space in which geometric object is considered. The basic idea is that one part of this system of equations is used for spatial imaging of the object, and the other part - for color imaging. Thus, the combined use of spatial and color imaging allows to reduce the dimension of the space, involved in spatial visualization, and it becomes possible to visualize the additional properties of the geometric object. A distinctive feature of the proposed approach is the use of a continuous non-linear color encoding information by using a continuous function to expand the imaging capabilities of geometric multidimensional space objects. The work provides 6 examples of the practical use of the proposed approach for the visualization of one-parameter and two-parameter geometric objects. At the same time, the possibilities of visualizing one-parameter objects by the example of arcs of algebraic curves belonging to spaces of different dimensions and two-parameter ones by the example of visualizing a portion of a topographic surface are investigated. The prospect of further research is to summarize the proposed approach for visualizing three-parameter geometric objects and bodies, as well as the reconstruction of three-dimensional geometric objects based on color images.
\end{abstract}

Keywords: BN-calculus, geometric object, coordinate-wise calculation, visualization, non-linear color coding.

\section{Introduction}

Most of the information about the world around a person is received through sight. In the process of visual perception of an object, researchers, above all, pay attention to its shape and color. The majority of computer-aided design and design systems are built on a similar property of human perception of information. This approach allows us to convey not only information about the shape of the test (or projected) object, but also additional properties as color support, reflecting the change in the physical, mechanical, architectural and other properties of the object. However, such a color reproduction of in-formation is either a discrete set of colored zones (e.g., computer-aided design systems, the finite element analysis [1-2]), or a linear relationship (e.g., geo-information systems with or without using a gradient [3] it [4]). 
In this paper, it is proposed to use continuous nonlinear color coding of information to expand the capabilities of visualizing multidimensional space, in which the color change is a certain continuous function.

For this, it is necessary that the analytical description of the multifactor process (or phenomenon) under study be presented in parametric form, in which each individual axis of the global coordinate system corresponds to only one specific characteristic of the simulated geometric object. A similar approach is used to visualize multidimensional geometric objects by projecting them on the projection plane [5]. Only in this case, projection does not occur on the plane, but on the axis of the projections.

The BN-calculus (the Balyuba-Naydysh calculus [6-8]) was developed in the 90 os of the 20th century by a team of scientists from the Melitopol School of Applied Geometry. By origin, $\mathrm{BN}$-calculus is a synthesis of vector, tensor, and barycentric calculi, from which it borrowed ideas and methods for determining geometric objects at the stage of formation, and their analytical description [6]. Initially, the BN-calculus was created as a special mathematical apparatus for engineering calculations related to modeling curves and surfaces of any shape in accordance with predetermined requirements and in the required parameterization. However, in the process of its development, in addition to shaping geometric objects, it found wide application in the field of modeling and optimization of multifactor processes and phenomena using multidimensional interpolation and approximation in their geometric interpretation [9-11].

The basic element of the BN-calculus is a point, and all geometric objects are defined as an organized set of points, for the analytical description of which the invariant properties of the parameter with respect to parallel projection are used. Proceeding from this, the BN calculus in the affine space can be considered a special case of the Wurf calculus in the projective space proposed by H. Staudt.

The main distinguishing feature of the $\mathrm{BN}$ calculus is that each projection of the desired geometric object on the axis of the global Cartesian coordinate system is determined separately. And the geometric object itself is the result of the joint interaction of all projections. Thus, using the color scheme, it becomes possible, along with the geometric shape, to convey some additional properties that correspond to one of the axes of the global coordinate system.

It should be noted that the selection of one of the parameters using color coding is a fairly common technique for constructing a visual representation, which is an effective tool for scientific visualization for systematization, modeling and analysis of multidimensional data $[12-13]$.

\section{Coordinate-wise calculation of geometric objects in the BN-calculus}

The point equations inherently represent a specific symbolic record, which includes a collection of simplex points and some functions of the current and fixed parameters. For the practical use of point equations, it is necessary to determine the starting points in a certain coordinate system. Such a procedure in the BN-calculus was called the coordinate-wise calculation, the result of which is a system of the same type of parametric equations. Moreover, each of the parametric equations is geometrically a projection of the desired geometric object onto one of the axes of the global coordinate system. Coordinate-wise calculation is one of the key elements of the theory of $\mathrm{BN}$ calculus and its essence is easiest to consider with a simple example.

Let two points be given: $A\left(x_{A}, y_{A}, z_{A}, \cdots\right), B\left(x_{B}, y_{B}, z_{B}, \cdots\right)$. The equation of the line has the form: $\frac{x-x_{A}}{x_{B}-x_{A}}=\frac{y-y_{A}}{y_{B}-y_{A}}=\frac{z-z_{A}}{z_{B}-z_{A}}=\cdots$. It is easy to notice the remarkable peculiarity of this formula - the identity of the expressions between the signs of equality, regardless of the 
dimension of space. If the constant ratio of the difference in the coordinates of the points is taken as a parameter $t$ :

$$
\frac{x-x_{A}}{x_{B}-x_{A}}=\frac{y-y_{A}}{y_{B}-y_{A}}=\frac{z-z_{A}}{z_{B}-z_{A}}=\cdots=t
$$

and then enter a convenient character notation for this coordinate expression system: $\frac{M-A}{B-A}=t$, then we get a compact notation of the equation of a straight line in point form.

We note that the system of coordinate-wise equations allows one to perform algebraic operations in point form:

$$
\frac{M-A}{B-A}=t \leftrightarrow M=(B-A) t+A .
$$

Moreover, all arithmetic operations are performed with points as with ordinary numbers. The last point equation defines a point $A$ at the value $t=0$, point $B-$ at $t=1$, line segment $A B-$ at $0 \leq t \leq 1$. We conclude that the point equation of the segment is provided by a special selection of the parameter. Obtaining required points using point equations determines the mathematical apparatus of BN calculus. In this case, the parameter should ensure the identity of mathematical operations with points, i.e. with their coordinates. Since the graphic image of a point in a Cartesian coordinate system is based on parallel projection, the invariant of such a projection should be a parameter - a simple ratio of three points of a line.

For example, moving from a point equation of a straight line to a system of parametric equations, for three-dimensional space we get:

$$
M=(B-A) t+A \rightarrow\left\{\begin{array}{l}
x_{M}=\left(x_{B}-x_{A}\right) t+x_{A} \\
y_{M}=\left(y_{B}-y_{A}\right) t+y_{A} \\
z_{M}=\left(z_{B}-z_{A}\right) t+z_{A}
\end{array}\right.
$$

Moreover, the number of parametric equations directly depends on the dimension of the space in which the desired geometric object is located.

Similarly, replacing the points with the coordinates corresponding to them, a transition is made from symbolic point equations to a system of parametric equations.

The use of a system of parametric equations for visualizing geometric objects is more preferable since it allows not only to unambiguously determine the type of geometric object by a parameterized attribute, but also to effectively use separate equations corresponding to projections on the axis of the global coordinate system for coding of various properties of the simulated object.

\section{Features of visualization of flat lines}

In geometric modeling of multifactor processes and phenomena [9-11], a curved line, as a one-parameter set, serves as an analytical description of one-factor processes and phenomena. It should be noted that a straight line is a special case of a curve of a line with zero curvature. Moreover, for the visual representation of a straight line, it is sufficient to have one-dimensional space just by this line both formed and limited to a certain segment. For graphical visualization of a curved line, it is necessary to use at least two-dimensional space - a plane, in case of a flat curve (visualization of spatial curves is discussed below). However, if you use color coding of a curve line, you can limit yourself to one-dimensional space. Then the problem is reduced to representing a straight line on which the color changes with a change in curvature. The easiest way to organize such a visualization is to use separate projections of a flat curve of a line on the axis of a flat Cartesian coordinate system, the analytical reflection of which is a system of parametric equations.

Let us consider an example of visualization of a plane parabola passing through 3 predetermined points [14]. The point equation of a parabola has the following form: 


$$
M=M_{1} \bar{t}(1-2 t)+4 \bar{t} t M_{2}+M_{3} t(2 t-1),
$$

where $M$ - current point of parabola arc;

$M_{i}$ - points through which the parabola arc passes;

$t$ - current parameter that varies from o to 1 ;

$\bar{t}=1-t-$ padding the parameter to 1 .

Passing from the point equation to the parametric, we obtain:

$$
\left\{\begin{array}{l}
x_{M}=x_{M_{1}} \bar{t}(1-2 t)+4 \bar{t} t x_{M_{2}}+x_{M_{3}} t(2 t-1) ; \\
y_{M}=y_{M_{1}} \bar{t}(1-2 t)+4 \bar{t} t y_{M_{2}}+y_{M_{3}} t(2 t-1) .
\end{array}\right.
$$

The visualization of such a curve in the plane will have the following form (Fig. 1).

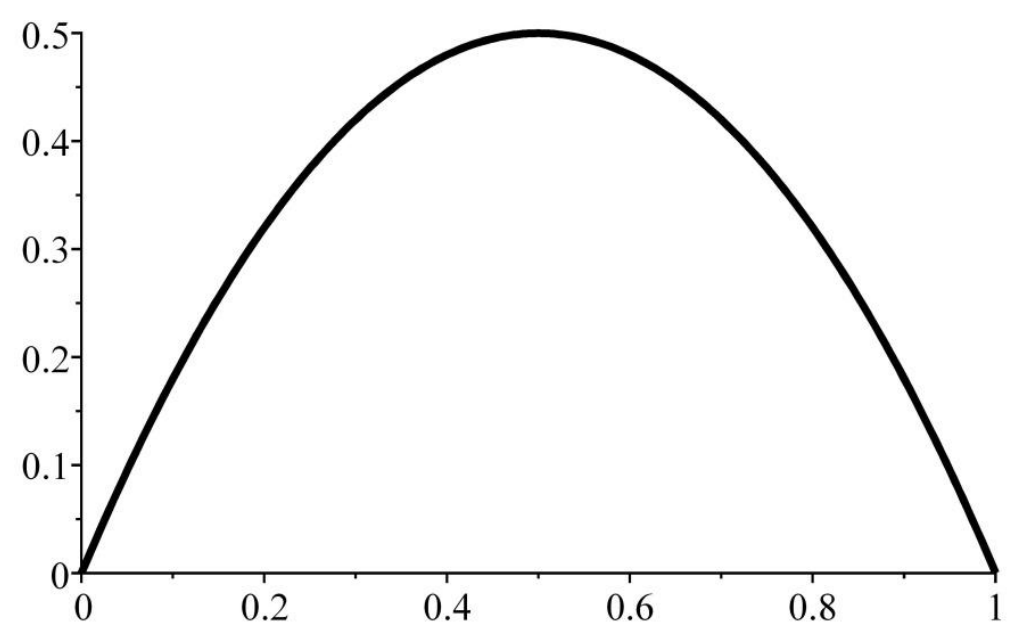

Fig. 1. Visualization of a flat parabola

Next, we construct a line segment $M_{1} M_{3}$, whose color will change depending on the position of the current point. So, the movement of the current point will be determined by the equation $x_{M}=f(t)$, and the color of the segment for each position of the current point $y_{M}=\psi(t)$. As a result, we obtain the following visualization of the parabola arc (Fig. 2), for which in order to restore the values of the function $y_{M}=\psi(t)$ you can use the attached color legend in the form of a spectrogram.

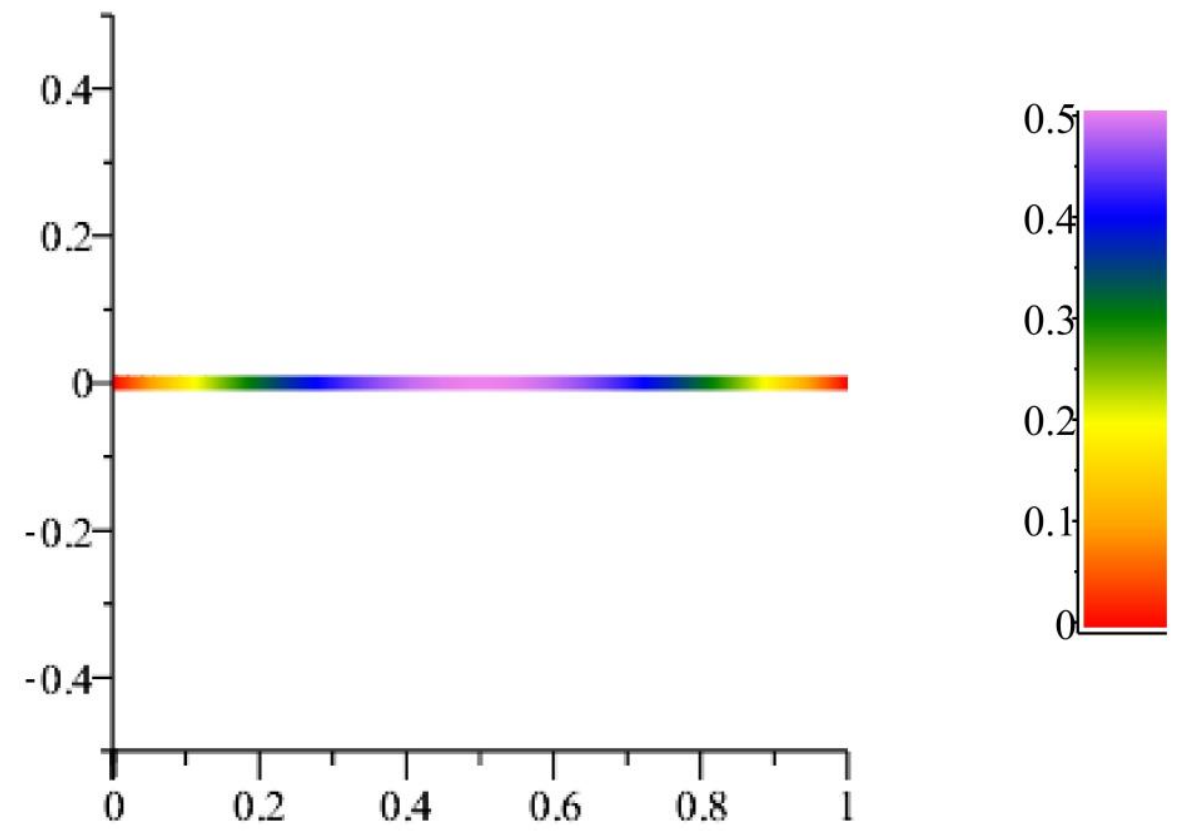

Fig. 2. Visualization of a parabola in which the coordinate change is color coded 
It should be noted that the obtained color segment of the straight line is a projection of the parabola arc, the maximum of $y=0,5$, which is achieved at a value that corresponds to the violet color. Moreover, Figure 2 clearly shows how the curve first increases to the maximum point, and then decreases.

Thus, the use of color coding allowed us to reduce the dimensionality of the space of the geometric object by one during the visualization process.

\section{Features of visualization of spatial lines}

Modeling of spatial lines which have more degrees of freedom compared to flat lines has its own distinctive features. The term "spatial curve" is usually understood as a line of double curvature, the points of which do not lie in the same plane. In a sense, this definition is contradictory and holds true exclusively for 3-dimensional space. For example, for a curve of the 4th order, which generally belongs to 4-dimensional space, the points also will not lie in the same plane, moreover, they will not lie in the same 3-dimensional space.

The same is true for algebraic curves of $n$-order. However, to fully visualize the shape of spatial curves, you can only imagine space of dimension $n$, which is an extremely difficult task. Therefore, to reduce the dimension of space, the projection method is used, most often orthogonal. Thus, any spatial curve can be represented as a set of projections on the projection plane of the global coordinate system, which is not very convenient for analytical calculations, for which projection is not preferred on the projection plane, but on the axis of the global coordinate system .

When considering algebraic curves of the $n$-order, it should also be taken into account that they are a collection of $n-1$ sets existing in spaces of various dimensions. For example, a third-order curve can be represented in the form of two sets [15]: a flat curve (cube) and a spatial curve (belonging to a 3 -dimensional space).

Now the visualization of spatial lines is carried out by means of computer graphics, mainly by approximation. For example, the work [16] gives an example of the capabilities of the 3dMAX visual modeling program that allows you to visualize a spatial curve by skipping circular sections along it.

Better visualization results can be obtained using color coding of one of the coordinates of the system of parametric equations of the spatial curve. At the same time, computer mathematics systems are used to visualize spatial curves. This approach allows us to increase the dimension of the space for visualizing algebraic curves of higher orders.

Consider an example of visualization of a third-order spatial Bezier curve in the form of a flat projection onto the horizontal plane of projections, which is determined by the following point equation:

$$
M=A_{1} \bar{t}^{3}+3 A_{2} \bar{t}^{2} t+3 A_{3} \bar{t} t^{2}+A_{4} t^{3},
$$

where $M-3$ rd arc Bezier curve current point;

$A_{i}$ - points determining the position of the tangents $A_{1} A_{2}$ and $A_{3} A_{4}$ 3rd order Bezier arcs.

Having performed the coordinate-wise calculation of the point equation of the arc of a third-order Bezier curve in a 3-dimensional space, we obtain:

$$
\left\{\begin{array}{l}
x_{M}=x_{A_{1}} \bar{t}^{3}+3 x_{A_{2}} \bar{t}^{2} t+3 x_{A_{3}} \bar{t} t^{2}+x_{A_{4}} t^{3} \\
y_{M}=y_{A_{1}} \bar{t}^{3}+3 y_{A_{2}} \bar{t}^{2} t+3 y_{A_{3}} \bar{t} t^{2}+y_{A_{4}} t^{3} \\
z_{M}=z_{A_{1}} \bar{t}^{3}+3 z_{A_{2}} \bar{t}^{2} t+3 z_{A_{3}} \bar{t} t^{2}+z_{A_{4}} t^{3}
\end{array}\right.
$$



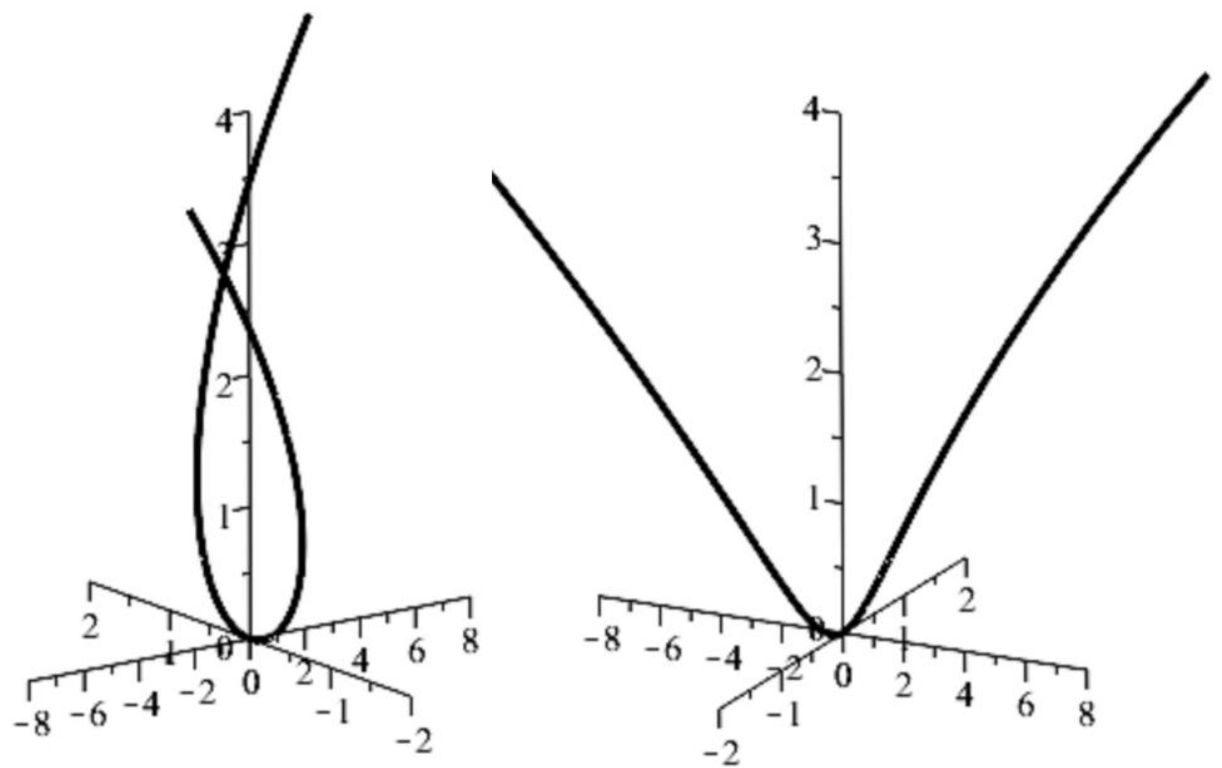

Fig. 3. Visualization of the arc of the curve of the 3rd order in 3-dimensional space

In fig. 3 spatial arc of a curve of the 3rd order is shown from different angles. Such a need arises, since in a static image from one angle it is not obvious that the arc of the curve is a line of double curvature, and not a flat line.

We use a combination of functional dependencies of the coordinates $x$ and $y$ of the current point $M$ to determine the position of the horizontal projection of the spatial curve, and set the elevation ( $M$ point's $z$ coordinate) by changing the color palette. The results of visualization of the spatial Bezier curve of the 3rd order are presented in Fig. 4.
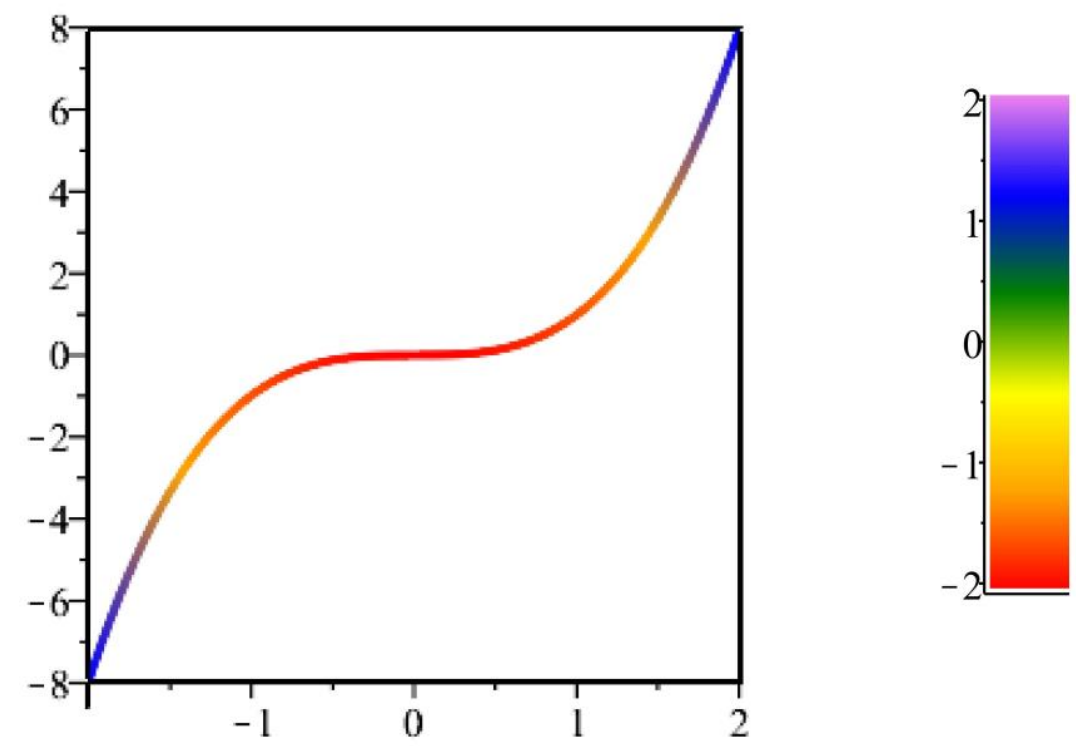

Fig. 4. Visualization of the spatial curve of double curvature on the plane

Color coding can also be used to enhance the effect, to emphasize some properties of the simulated object. For example, Figure 5 shows the visualization of a spatial curve for which the elevation values duplicate the change in the color of the curve arc along the current point. 


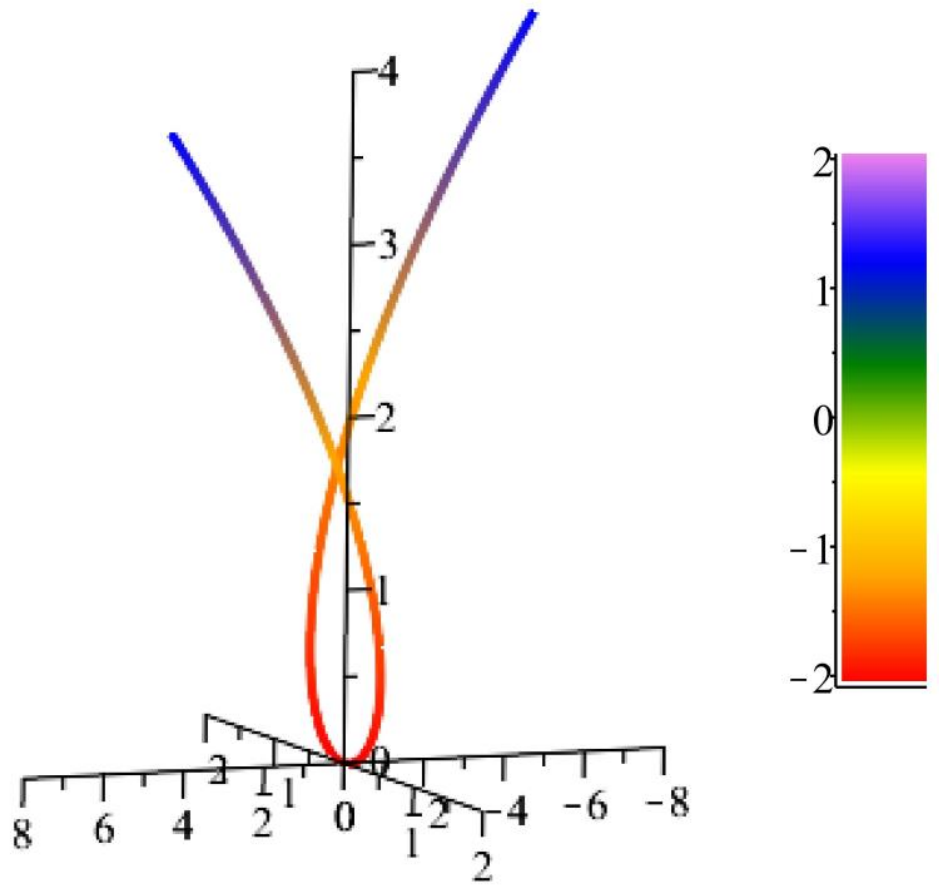

Fig. 5. Visualization of a curve of double curvature in 3-dimensional space

The proposed approach to the visualization of spatial lines can be generalized and used to visualize spatial curves belonging to 4-dimensional space. Of algebraic curves, curves starting from the fourth order possess the necessary properties.

Consider an example of visualization of an arc of a fourth-order curve passing through the 5 ahead given points [17] in 4-dimensional space:

$$
M=M_{1} p_{1}+M_{2} p_{2}+M_{3} p_{3}+M_{4} p_{4}+M_{5} p_{5},
$$

where $p_{1}=\bar{t}^{4}-\frac{13}{3} \bar{t}^{3} t+\frac{13}{3} \bar{t}^{2} t^{2}-\bar{t} t^{3}$;

$p_{2}=16 \bar{t}^{3} t-\frac{64}{3} \bar{t}^{2} t^{2}+\frac{16}{3} \bar{t} t^{3} ;$

$p_{3}=-12 \bar{t}^{3} t+40 \bar{t}^{2} t^{2}-12 \bar{t} t^{3}$;

$p_{4}=\frac{16}{3} \bar{t}^{3} t-\frac{64}{3} \bar{t}^{2} t^{2}+16 \bar{t} t^{3} ;$

$p_{5}=-\bar{t}^{3} t+\frac{13}{3} \bar{t}^{2} t^{2}-\frac{13}{3} \bar{t} t^{3}+t^{4}$.

The system of parametric equations of the arc of a 4th-order curve in 4-dimensional space will have the following form:

$$
\left\{\begin{array}{l}
x_{M}=x_{M_{1}} p_{1}+x_{M_{2}} p_{2}+x_{M_{3}} p_{3}+x_{M_{4}} p_{4}+x_{M_{5}} p_{5} ; \\
y_{M}=y_{M_{1}} p_{1}+y_{M_{2}} p_{2}+y_{M_{3}} p_{3}+y_{M_{4}} p_{4}+y_{M_{5}} p_{5} ; \\
z_{M}=z_{M_{1}} p_{1}+z_{M_{2}} p_{2}+z_{M_{3}} p_{3}+z_{M_{4}} p_{4}+z_{M_{5}} p_{5} ; \\
r_{M}=r_{M_{1}} p_{1}+r_{M_{2}} p_{2}+r_{M_{3}} p_{3}+r_{M_{4}} p_{4}+r_{M_{5}} p_{5} .
\end{array}\right.
$$

In accordance with the proposed visualization method, we use the first three equations from the system of parametric equations to determine the position of the current point in 3-dimensional space, and we can represent the fourth equation by changing the values of the color palette. As a result, we obtain a visualization of one of the projections of the spatial arc of a 4th-order curve belonging to 4-dimensional space (Fig. 6). 

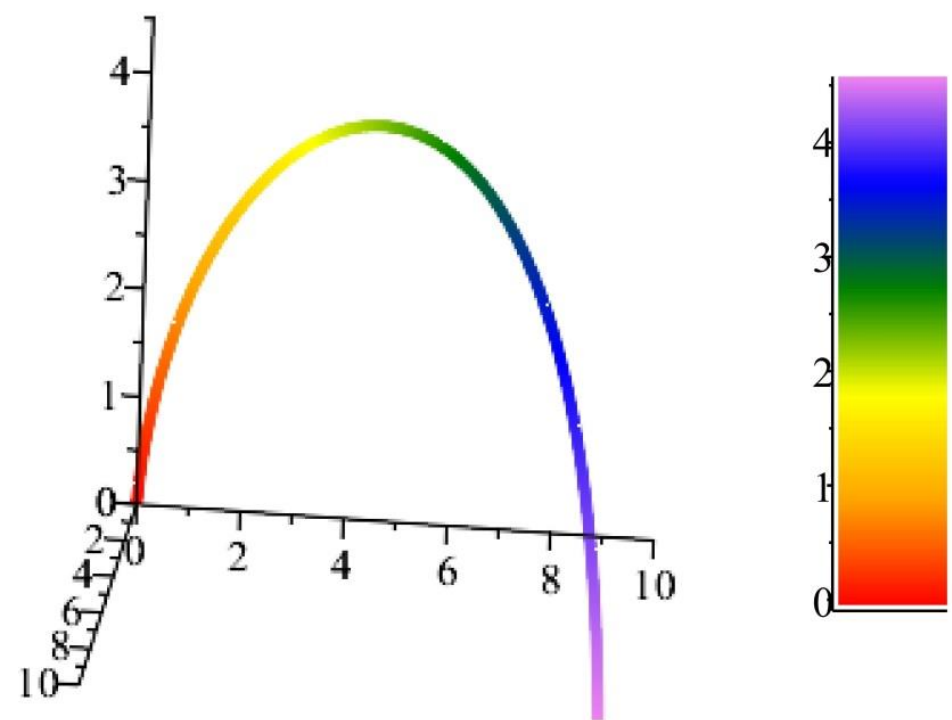

Fig. 6. Visualization of the projection of the spatial arc of a 4th-order curve on 3-dimensional space

Of course, not all spatial lines are conveniently visualized in this way. For example, visualization of a helical cylindrical line will not be possible, since the projections of the current point of the curve will overlap each other. In many cases, the proposed visualization method can serve as an effective tool for acquiring new knowledge about the geometric properties of objects in 4-dimensional space.

\section{Features of visualization of surface compartments}

Visualization of the surface is not a trivial task in itself, since it requires the placement of a three-dimensional image in the plane of the screen. Axonometric and perspective projections, discretization, color change, and other visualization technologies are used here. Moreover, the surface itself can be represented as a set of discrete points, lines, triangles, rectangles and other elements. A separate type of visualization, including surfaces, are nomograms, which are widely used in engineering practice and represent a discrete set of lines with certain properties that usually correspond to fixed values of one of the factors under study. In this case, nonlinear grids (for example, logarithmic) are often used.

One of the ways to visualize such nomograms can be by using color coding of one of the coordinates, which also allows to reduce the dimensionality of the space for analyzing the results.

As an example, let us consider a visualization of a two-factor process of the dependence of heat $Q$ received from a boiler unit on the temperature head of a heat carrier $t$ and the diameter of convective pipes $d$ (Fig. 7). 


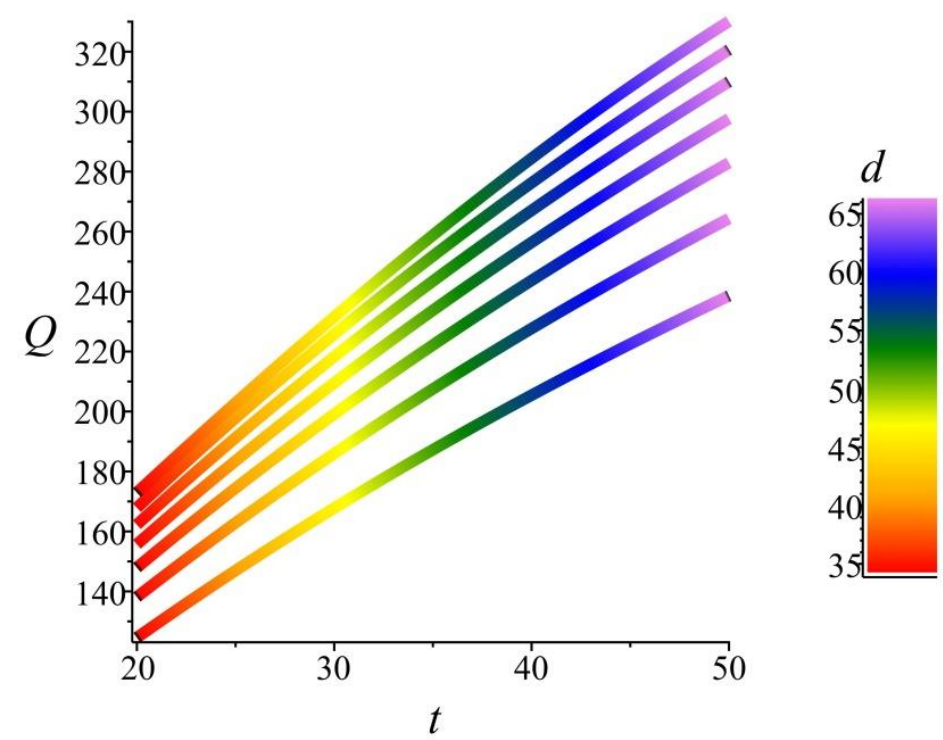

Fig. 7. Visualization of a two-factor process in the form of a nomogram

Another, more effective way of visualizing the surface compartment using color coding is to use not the discrete lines shown on the nomogram, but a continuous color field of values (Fig. 8).
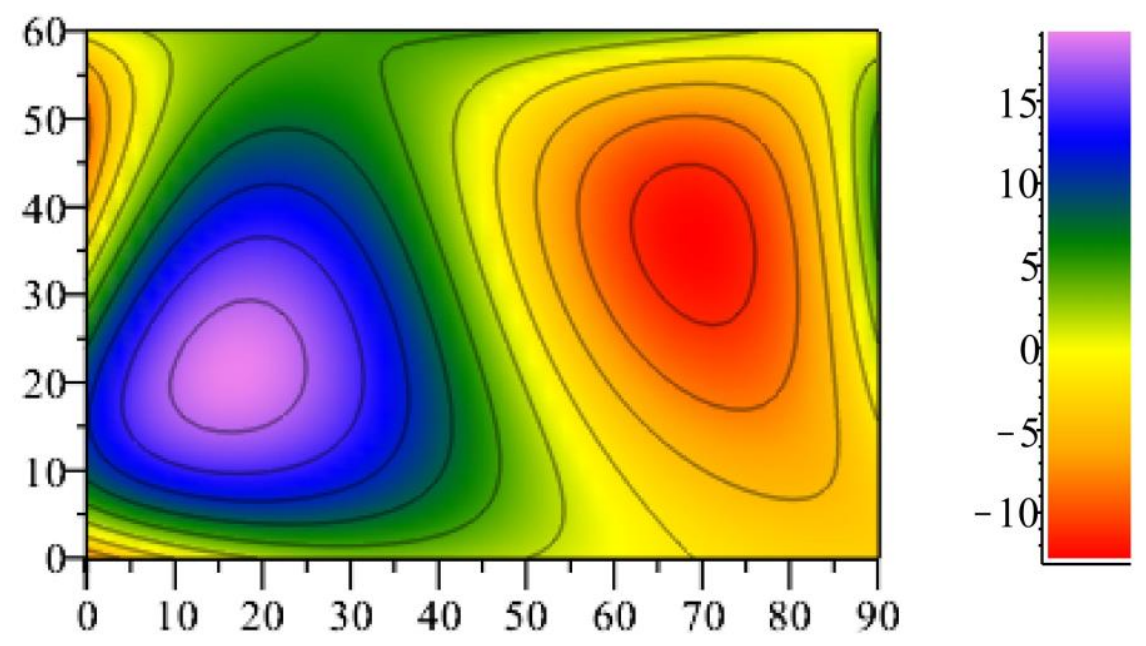

Fig. 8. Visualization of a plot of a topographic surface using color coding of a coordinate change $z$

Figure 8 shows an example of visualization of a section of a topographic surface, the geometric model of which is presented in [18], using color coding of changes in elevations corresponding to changes in coordinates $z$.

A similar visualization method is widespread in the two-dimensional problems of computational physics to represent the studied quantity as a function of two spatial variables. For example, in [19-20].

\section{Conclusion}

The paper proposes an approach that allows visualization of geometric objects based on the point equations of their formation. This approach allows for visualization to reduce the dimension of the space, which, in future, makes it possible to visualize more properties that characterize the projected or investigated object. The given examples confirm the possibility of using the proposed approach in engineering and scientific practice for visualization. 
Of course, like any projection apparatus, color coding of coordinate changes has its drawbacks. The main one is the complexity of representing a geometric object in space, which requires the user to have an advanced spatial thinking skill. Nevertheless, it can be effectively used as one of the alternative tools for engineering and scientific visualization, which has its own advantages.

The prospect of further research is visualization using the point equations of geometric bodies and hypersurfaces as a multi-parameter set of points belonging to a multidimensional space.

\section{References}

1. Tolmacheva YU.P., Shmatkova A.V., Zajdes S.A. The use of the finite element method for visualizing the stress-strain state of fish bones // Scientific Visualization 11.2: 114125, 2019. DOI: 10.26583/sv.11.2.09. [in Russian].

2. Sergeev D.A., Ermakova O.S., Suvorov A.S., Troickaya Yu.I., Kandaurov A.A., Kon'kov A.I [The use of visualization tools to verify methods of numerical modeling in a reference experiment around a rectangular obstacle flow // Scientific Visualization 10.2: 114, 2018. DOI: 10.26583/sv.10.2.01. [in Russian].

3. Stepanov YU.A., Burmin L.N., Stepa-nov A.V. Visualization of sound frequencies of the flow of geomechanically processes using GIS technologies // Scientific Visualization 10.3: 144-151, 2018. DOI: 10.26583/sv.10.3.11. [in Russian].

4. Belyaev K.P., Kuleshov A.A., Tuchko-va N.P. Estimates of the meridional heat and mass transfer in the South Atlantic based on a joint model of atmospheric and ocean circulation with assimilation of observational data and using visualization tools // Scientific Visualization 11.1: 119-138, 2019. DOI: 10.26583/sv.11.1.10. [in Russian].

5. Barabanov V.F., Nuzhnyj A.M., Pod-val'nyj S.L., Safronov V.V. Development of a software complex for restoration and visualization of $3 \mathrm{~d}$ models for a set of approximate orthogonal projections // Scientific Visualization, 2017. Vol.9. №2. pp. 82-93. [in Russian].

6. Balyuba I.G., Goryagin B.F., Malyu-tina T.P., Davydenko I.P., Konopatskiy E.V. Point calculus of geometric forms and its place among other existing calculi // Computerintegrated technologies: education, science, production. Lutsk: LNTU, 2011. №6. pp. 2429. [in Russian].

7. Najdysh V.M. Balyuba I.G., Vereshchaga V.M. Algebra of BN-Calculus // Applied geometry and engineering graphics. Kiev: KNUBA, 2012. Vol. 90. pp. 210-215. [in Russian].

8. Bumaga A.I., Konopatskiy E.V., Krys'ko A.A., Chernysheva O.A. Introduction to the mathematical apparatus of BN calculus // Materials of the VII International scientific and practical Internet conference "Problems of quality of graphic training of students in a technical University: traditions and innovations". Perm: PNIPU, 2017. Vol. 4. pp. 76-82. [in Russian].

9. Konopatskiy E.V. An approach to the construction of geometric models of multifactor processes and the phenomena of multidimensional interpolation // Software engineering. Moscow: 2019. Vol.10. № 2. pp. 77-86. [in Russian].

10. Konopatskiy E.V. Geometric modeling and optimization of multifactor processes and phenomena by multidimensional interpolation // Proceedings of the International scientific conference on physical and technical Informatics CPT-2018, may 28-31, 2018. Moscow-Protvino, 2018. pp. 299-306. [in Russian].

11. Konopatskiy E.V. The principles of building computer models of multifactor processes and phenomena by the method of multidimensional interpolation // Collection of materials of the II International scientific and practical conference: "Software engineering: method and technologies for developing information and calculation systems (PIIVS2018)". November 14-15, 2018 Donetsk: DonNTU, 2018. pp. 277-287. [in Russian]. 
12. Zinov'ev A. YU. Multidimensional Data Visualization // Krasnoyarsk, Izd. KGTU, 2000. 180 p. [in Russian].

13. Bondarev A.E., Galaktionov V.A. Analysis of multidimensional data in the tasks of multi-parameter optimization using visualization methods // Scientific Vizualisation, 2012. Vol. 4, № 2. pp. 1-13. [in Russian].

14. Bumaga A.I. 2-nd order parabola arc equation // Applied geometry and engineering graphics. Kiev: KNUBA, 2012. Vol. 90. pp. 49-51. [in Russian].

15. Glagolev N.A. Projective geometry // Moscow: Higher school, 1963. 344 p. [in Russian].

16. Ivashchenko A.B., Petrova L.A. Using 3dMAX to study spatial curves // MGSU Bulletin, 2011. №2/2011. pp. 358-360. [in Russian].

17. Konopatskiy E.V. Modeling arcs of curves passing through predetermined points // Moscow: 2019. № 2. pp. 30-36. DOI: 10.14489/vkit.2019.02.pp.030-036. [in Russian].

18. Konopatskiy E.V., CHernysheva O.A., Kokareva YA.A. Modeling a curved section of a topographic surface on an irregular network of points // Bulletin of computer and information technologies. Moscow: 2018. № 7. pp. 17-22. DOI: 10.14489/vkit.2018.07.pp.017022. [in Russian].

19. Bondarev A.E., Galaktionov V.A., Chechetkin V.M. Analysis of the Development Concepts and Methods of Visual Data Representation in Computational Physics. Computational Mathematics and Mathematical Physics, 2011. Vol. 51. No. 4. pp. 624-636.

20. Bondarev A.E. On visualization problems in a generalized computational experiment (2019). Scientific Visualization 11.2: 156-162. DOI: 10.26583/sv.11.2.12. 